\title{
SAR Shape from Shading in suburban areas
}

\author{
Gerardo Di Martino, Alessio Di Simone, Antonio Iodice, Daniele Riccio, Giuseppe Ruello \\ Dipartimento di Ingegneria Elettrica e delle Tecnologie dell'Informazione \\ Università di Napoli Federico II, 80125, Napoli, Italy \\ \{gerardo.dimartino, alessio.disimone, antonio.iodice, daniele.riccio, giuseppe.ruello\}@unina.it
}

\begin{abstract}
Shape-from-shading (SfS) techniques can be applied to synthetic aperture radar (SAR) images in order to obtain estimates of the slopes and, then, the elevation of the observed scene. Recently the authors proposed a new SfS technique for natural scenarios, based on the use of fractal surface models: problems can arise if non-natural features are present in the observed scene. In this paper, we evaluate the impact of manmade structures on the technique's performance: in particular, the SfS algorithm is applied on a SAR image in which a suburban environment is present. A deep analysis of the main issues is performed and possible solutions are presented and discussed.
\end{abstract}

\section{INTRODUCTION}

The shape-from-shading problem consists in the reconstruction of the 3D shape of an object given a single 2D gray-level intensity map of it. Since the first seminal work by van Diggelen [1], shape from shading has experienced an increasing success in the research community attracting more and more researchers, especially in the computer vision field, where very accurate algorithms have been developed [2], [3]. The reason for this success has to be searched in the behavior of the human visual system, which, proficiently perceiving the three-dimensional structure of an object from its 2D shading pattern, shows a great analogy with SfS concepts. Most of the computer vision literature dealing with shape-from-shading techniques is based on a Lambertian law for the description of scattering at optical frequencies, due to its simplicity and good fitting with theoretical models and empirical experiments.

Extension of SfS principles to Synthetic Aperture Radar is not straightforward at all: with respect to optical systems and images usually used in computer vision literature, SAR systems and images are subject to different phenomena (scattering mechanisms, SAR range-based image acquisition) and present peculiar aspects, as the well-known geometrical distortions (foreshortening and layover). As a consequence, very little has been done in SfS applied to SAR images, and, moreover, most of the literature solves the scattering issue, "extending" the Lambertian model at microwave frequencies, where SAR systems usually operate. Starting from these considerations, the authors have shown that properly strengthening the modelization step, it is possible to overcome the limits of the state of the art [4]. In particular, they proposed a SfS technique based on the fractal geometry and suitable for natural surfaces. The proposed technique is able to provide raw estimates of the slopes and elevation of the observed natural surface. However, when manmade objects are present on the scene problems arise in the estimation.
In this paper, the impact of manmade structures on SfS technique's performance is evaluated. An actual Cosmo/SkyMed stripmap image of the Vesuvius volcano (close to Naples, Italy) is considered: hence, the scenario is made up of a large natural scene with the presence in some built-up areas. An analysis of the obtained results is performed and possible solutions aimed at mitigating the detrimental effects due to the presence of manmade features are proposed.

\section{SFS AND THE URBAN ENVIRONMENT}

Nowadays, everywhere urban areas are increasingly growing, so that most SAR applications designed for natural landscapes have to face the potential presence of (sub)urban settlements and man-made structures on the observed scene. Furthermore, the increased resolution of the available SAR imagery is the reason for the presence of more and more bright points in SAR images, especially in urban and suburban areas: this is due to multiple reflections and other scattering phenomena typical of built-up areas.

From the first SAR sensors, many 3D reconstruction and Digital Elevation Model (DEM) estimation techniques have been designed and improved (stereoscopy [5], interferometry [6], radarclinometry or SfS [7], polarimetry [8]), attesting an increasing interest in the knowledge about the shape of the Earth and other planets' surfaces. However, all of these techniques have been designed for natural landscapes and very poor performances are experienced in urban environments. SAR tomography [9], [10] seems to be a very promising technique for urban features extraction, also with highresolution data; the robustness and accuracy are paid by computationally expensive algorithms, need for huge datasets and the consequent impossibility to face with quick-response emergencies management. In this direction, simple and fast algorithms able to provide also approximate DEMs with few (even one) SAR images are desirable in order to operate even in a real-time operative mode. Among the aforementioned techniques, shape from shading has the main advantage to require only one intensity image so that multiple passes or complex image acquisition modes are no longer a strict requirement. The fractal model proposed by the authors [4] is aimed at recovering the topography under the hypothesis of a SAR image of a natural landscape; the direct model linking the SAR intensity map to the parameters of interest (range and azimuth slopes) is as follows [4] 


$$
\begin{gathered}
I(p, q)=G \Delta x \Delta r \pi(2 k)^{2(1-H)} S_{0}\left|\beta_{m n}\right|^{2}\left(p \sin \vartheta_{0}+\cos \vartheta_{0}\right)^{4} \\
\frac{\left(p^{2}+q^{2}+1\right)^{H-\frac{1}{2}}}{\left[\left(p \cos \vartheta_{0}-\sin \vartheta_{0}\right)^{2}+q^{2}\right]^{H+\frac{3}{2}}}
\end{gathered}
$$

where $G$ is a calibration constant, $\Delta x$ and $\Delta r$ are the azimuth and slant range pixel spacing respectively, $k$ is the propagation constant, $H$ is the Hurst coefficient, $S_{0}$ is the spectral amplitude, $\beta_{m n}$ is a coefficient depending on the range $(p)$ and azimuth $(q)$ local slopes and $\theta_{0}$ is the radar-look angle.

In order to simplify the inversion procedure, a linearization of the intensity function $I(p, q)$ is proposed:

$$
I=G\left(a_{0}+a_{1} p\right)
$$

A first step DEM is obtained integrating the estimated range slopes independently in the range direction. In order to reduce speckle effects and linage effect due to the independent integration and reintroduce azimuth-slope dependence, a MMSE regularization procedure is performed and the estimated DEM is obtained just integrating in the azimuth direction.

As shown in [4], the proposed technique provides satisfactory results, especially if compared with the widely used Lambertian model, if a SAR image of a natural surface is at hand. In presence of built-up areas, the fractal model experiences very poor performances, providing a totally wrong DEM. Note that this happens for every other SfS technique. This is due to the different mechanisms governing scattering phenomena in presence of man-made structures. Layover, shadowing, and multiple scattering from dihedral structures are very common in active remote sensing of urban areas, especially in high-resolution images, which are therefore characterized by a huge amount of very bright points. The proposed model does not take into account these kinds of phenomena, which are more uncommon in natural landscapes.

The main limit of the proposed SfS method is a great sensitivity against the calibration constant, whose estimation requires great care. Built-up areas can greatly affect the calibration step, just causing an overestimation of $G$ and then an underestimation of the range slope, except in built-up areas. As a consequence, the proposed technique provides unreliable results even in presence of small urban settlements, interpreting bright points as very steep slopes, as suggested by (2). Furthermore, the independent range integration together with a local azimuth regularization procedure, cause on the obtained DEM an error propagation in the range direction. Since range integration is performed from the middle range line, a far-range bright point is "translated" in a positive deep slope and vice versa for near-range bright points. Clearly, the propagation error does not affect the range slope estimation, that shows a good accuracy except in the suburban region, if calibration constant is well estimated in a built-up free region. This is a very interesting point, since, if urban settlements are not of interest, DEM estimation accuracy can be improved by a simple binary classification aimed at identifying manmade features. Also calibration constant estimation could greatly benefit from the classification step, thus limiting the estimation only to natural landscape. Thanks to the proper modelization step, a simple urban-non urban unsupervised classification can be performed. In this way, urban areas (and eventually layover points) are excluded from DEM estimation, consequently avoiding the error propagation effect. The proposed model is not valid in urban and suburban areas, then, for urban features extraction, properly models and techniques need to be developed in order to take into account the different scattering mechanisms.

For the sake of simplicity, in this paper we assume that built-up areas are not of interest (otherwise stated, Digital Terrain Model will be derived); the proposed modified fractal SfS technique is applied to a single-look stripmap Cosmo \SkyMed SAR image of the Vesuvius volcano, close to Naples, Italy (Fig. 1(a)). Urban settlements are clearly visible in the top and left side of the image. Figure 1(b) shows the ground-truth range slope map projected in the azimuth - slant range coordinate system. Figures 1(c) and (e) show the estimated range slope and elevation map provided by the technique proposed in [4]. Bright points in the SAR image due to the suburban areas cause gross errors in the estimated range slope map and then, a totally wrong elevation map, in which error propagation effects are clearly visible, is produced. In order to filter out suburban areas from DTM estimation, a simple binary classification based on intensity thresholding is performed and the results are shown in Fig. 1(d) and (f). Performance improvements are quantitatively evaluated through statistical parameters shown in Table I. In particular we have measured the median, mean and standard deviation of the absolute error on the elevation, range and azimuth slopes. As Fig. 1, Fig. 2 and Table I show, urban bright points are now dramatically reduced in the range slope estimation and DTM accuracy is greatly improved.

\section{CONCLUSION}

A study of the impact of the presence of manmade features on the scene on shape from shading has been carried out. If a DTM is of interest, built-up areas can be easily filtered out, thus significantly improving accuracy depending on the classification reliability. In this paper a simple binary classification based on intensity thresholding is performed in order to exclude urban areas from DTM estimation, experiencing good performance improvements. Better results will be provided with more sophisticated classification algorithms. Man-made structures height estimation cannot be performed without entering the different scattering issues typical of urban areas, properly adjusting the SfS model and the inversion technique. An appropriate model for urban settlements together with an interesting inversion technique somehow based on SfS principles and aimed at recovering 3D shape of built-up areas from a single SAR image can be found in [11]. In this direction, a binary classification-driven model selection algorithm could be set up in order to select the right model depending on the observed scene (natural or urban). 


\section{References}

[1] J. van Diggelen, "A photometric investigation of the slopes and the heights of the ranges of hills in the Maria of the moon," Bull. Astron. Inst. Netherlands, vol. 11, no. 243, pp. 283-290, 1951.

[2] I. Kemelmacher-Shlizerman, R. Basri, "3D Face Reconstruction from a Single Image Using a Single Reference Face Shape," IEEE Transactions on Pattern Analysis and Machine Intelligence, vol.33, no.2, pp. 394405, Feb. 2011

[3] D. Chen, F. Dong, "Shape from shading using wavelets and weighted smoothness constraints," IET Computer Vision, vol. 4, no. 1, pp. 1-11, March 2010.

[4] G. Di Martino, A. Di Simone, A. Iodice, D. Riccio, G. Ruello, "On shape from Shading and SAR Images: an Overview and a New Perspective," International Geoscience and Remote Sensing Symposium (IGARSS) 2014, pp. , 13-18 Jul. 2014.

[5] C. Pu-Huai, O. Clochez, and G. Saundercock I. Dowman, "Heighting from stereoscopic ERS-1 data," Proceedings Second ERS-1 Symposium, pp. 609-614, 1993

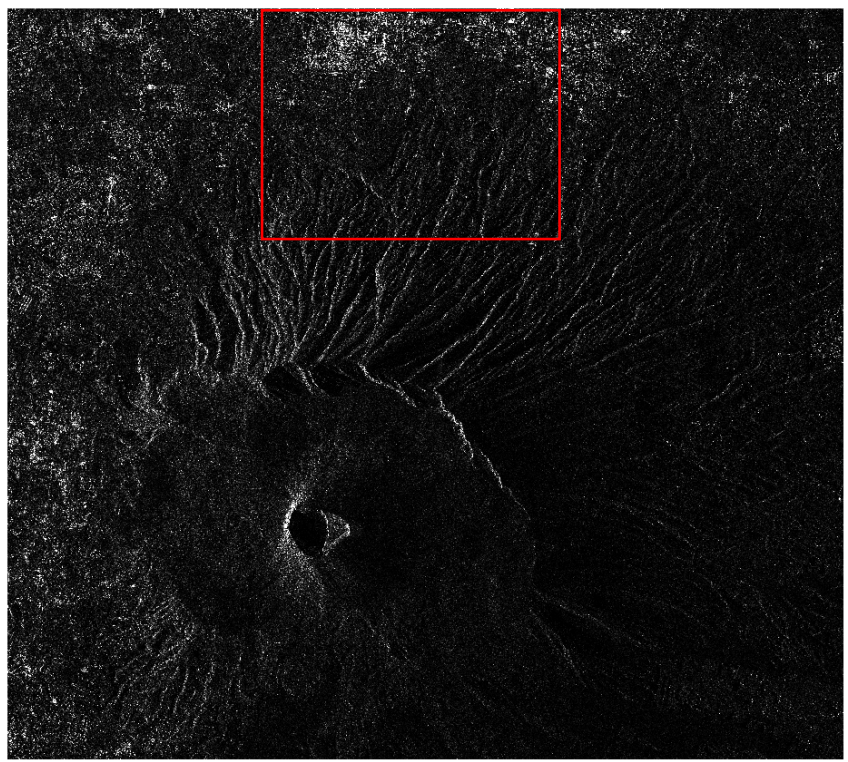

(a)

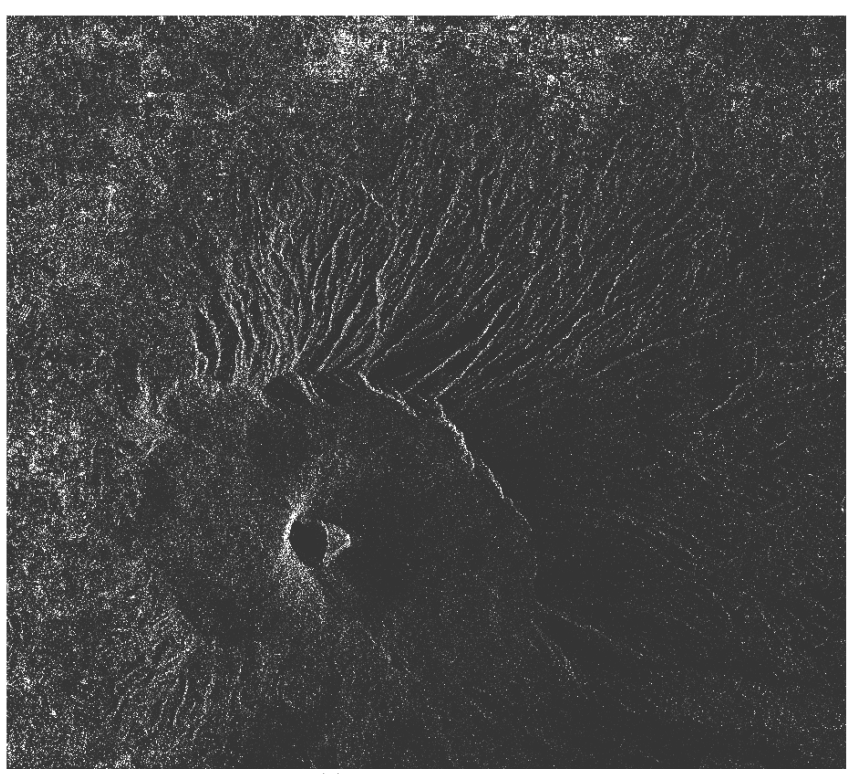

(c)
[6] D. Massonnet and T. Rabaute, "Radar interferometry: limits and potential," IEEE Trans. Geosci. Remote Sens., vol. 31, no. 2, pp. 455 464, 1993.

[7] D.N. Ostrov, "Boundary Conditions and Fast Algorithms for Surface Reconstructions from Synthetic Aperture Radar Data," IEEE Trans. Geosci. Remote Sens., vol. 37, no. 1, pp. 335-346, Jan. 1999.

[8] J. S. Lee, and G. De Grandi D. L. Schuler, "Measurement of topography using polarimetric SAR images," IEEE Trans. Geosci. Remote Sens., vol. 34, no. 5, pp. 1266-1277, Sep. 1996.

[9] X. X. Zhu and R. Bamler, "Very High Resolution Spaceborne SAR Tomography in Urban Environment," IEEE Trans. Geosci. Remote Sens., vol. 48, no. 12, pp. 4296-4308, 2010.

[10] G. Fornaro, A. Pauciullo, D. Reale, S. Verde, "Multilook SAR Tomography for 3-D Reconstruction and Monitoring of Single Structures Applied to COSMO-SKYMED Data," IEEE J. Sel. Top. Appl. Earth Obs. Remote Sens., vol. 7, no. 7, pp. 2776-2785, Jul. 2014.

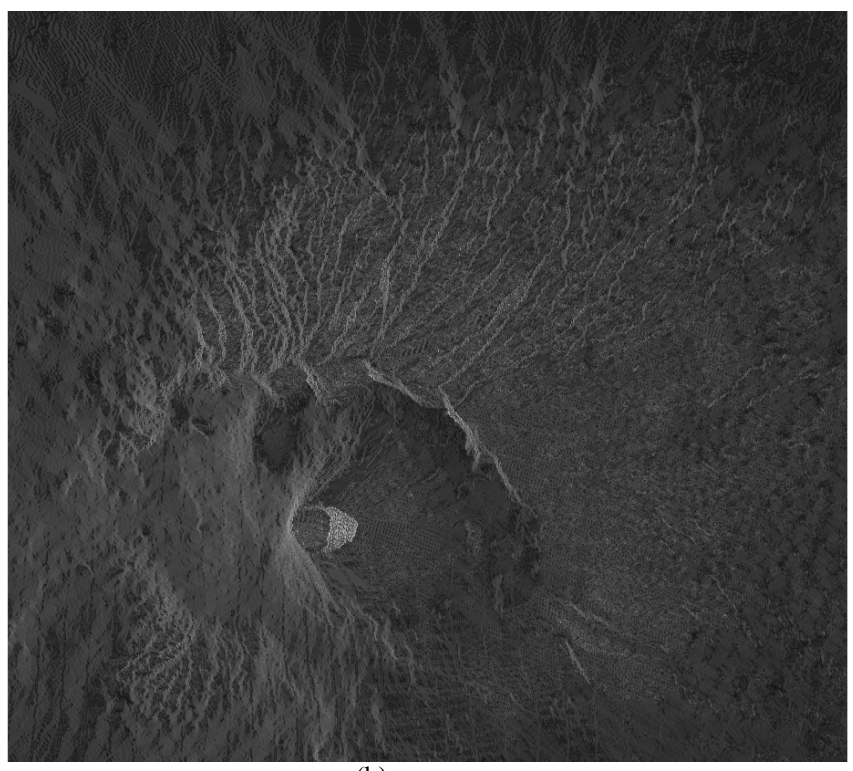

(b)

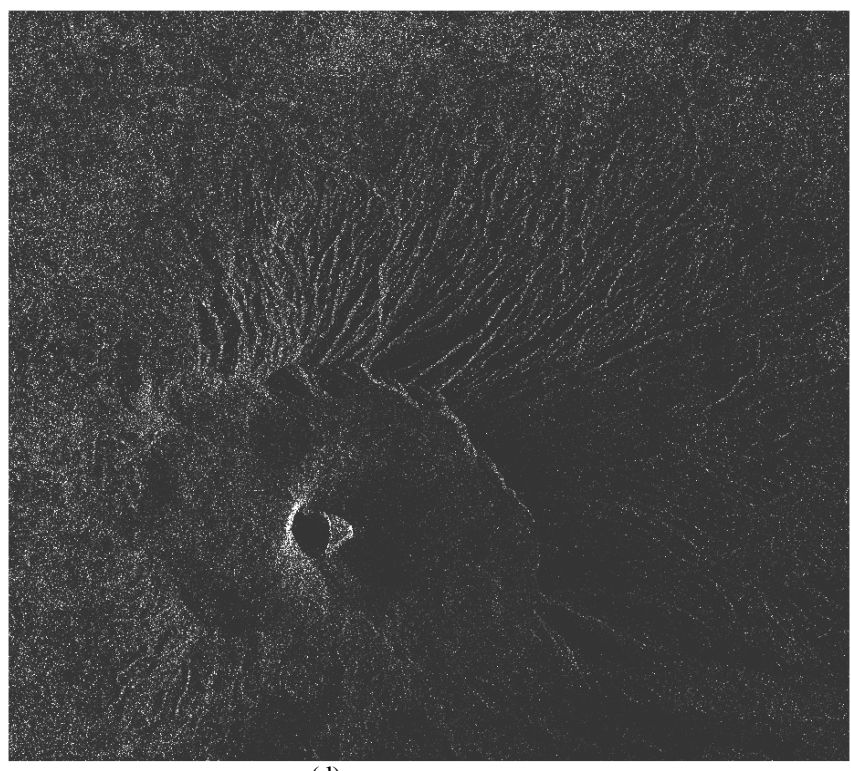

(d) 


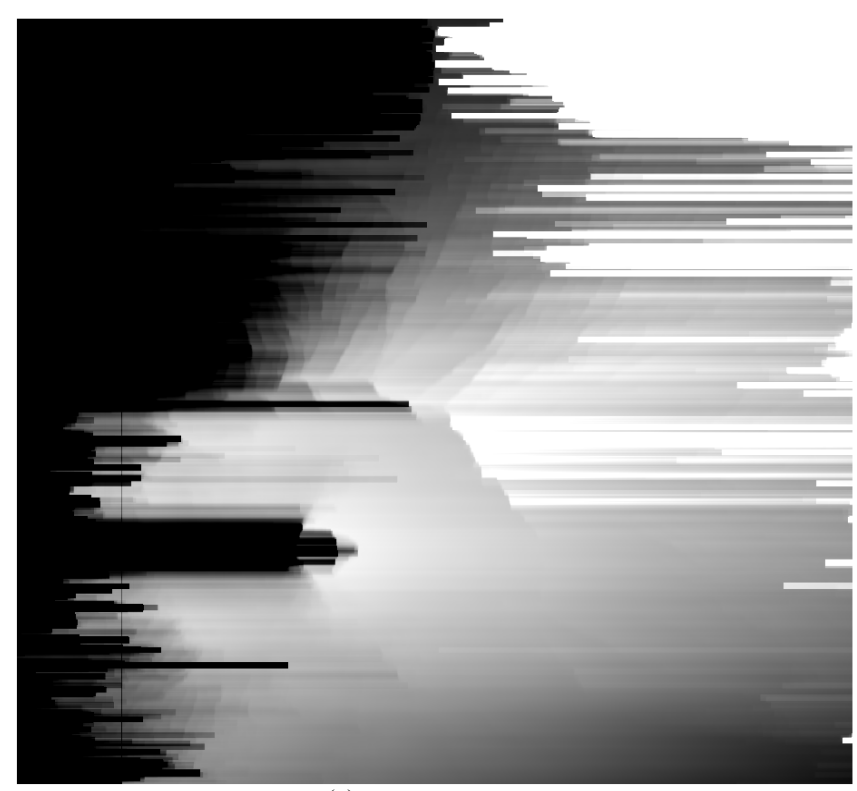

(e)

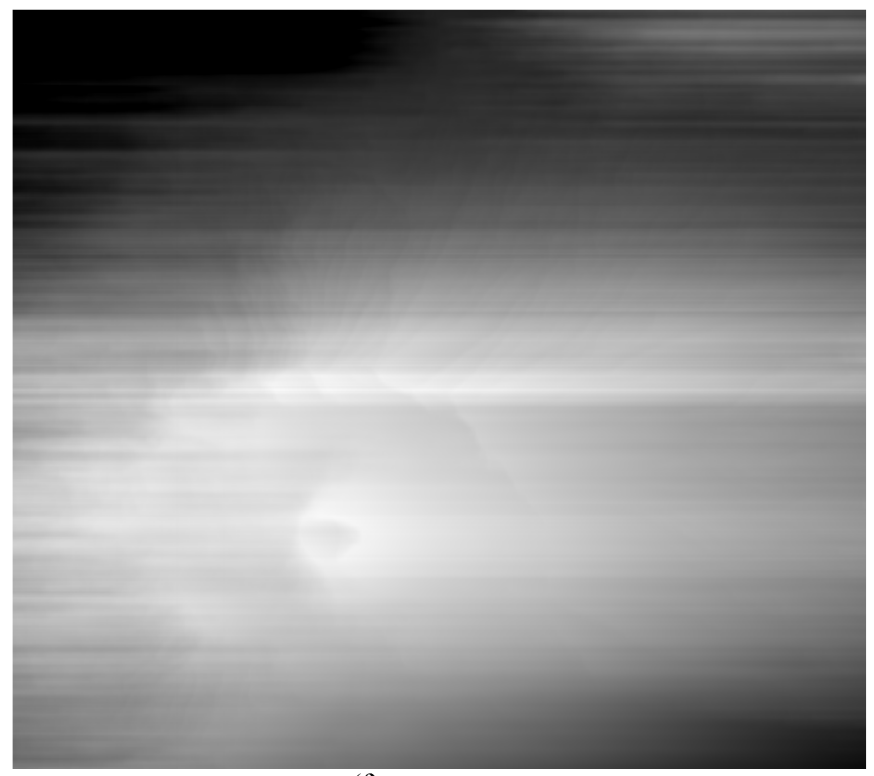

(f)

Fig. 1: (a) Single look CosmolSkymed SAR image; (b) ground-truth range slope map; (c) estimated range slope map without removing manmade features; (d) estimated range slope map removing manmade features; (e) estimated DEM without built-up areas masking; (f) estimated DEM with built-up areas filtered out. The red box in Fig. 1(a) identifies the zoomed region (see Fig. 2).

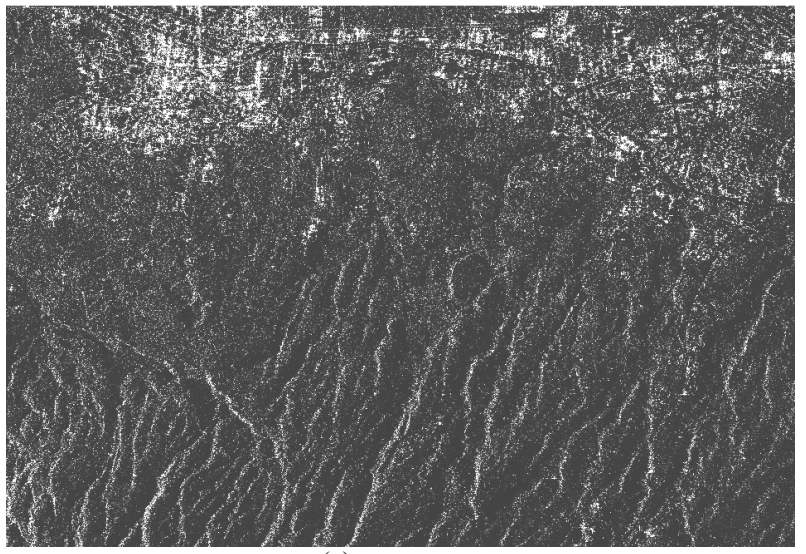

(a)

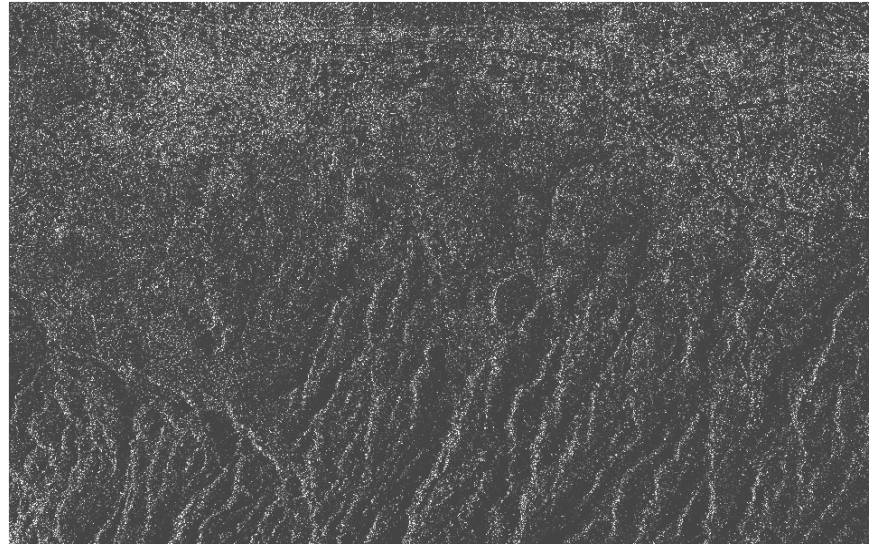

(b)

Fig. 2: Red box of Fig. 1(a) zoomed: estimated range slope without (a) and with (b) manmade structures filtering.

[11] G. Di Martino, A. Iodice, D. Riccio, and G. Ruello, "Power Spectra of Very High Resolution SAR Amplitude Images of Urban Areas," IEEE J.
Sel. Top. Appl. Earth Obs. Remote Sens., vol. 7, no. 7, pp. 2723-2731, Jul. 2014.

TABLE I

STATISTICAL PARAMETERS

\begin{tabular}{|c|c|c|c|c|c|c|c|c|c|}
\hline \multirow{2}{*}{ Error magnitude } & \multicolumn{3}{|c|}{ Altitude (m) } & \multicolumn{3}{|c|}{$\alpha_{\mathrm{r}}(\circ)$} & \multicolumn{3}{|c|}{$\alpha_{a}(\circ)$} \\
\hline & Median & Mean & Std dev. & Median & Mean & Std dev. & Median & Mean & Std dev. \\
\hline Without built-up areas masking & 344.9 & $3.205 \times 10^{4}$ & $1.100 \times 10^{5}$ & 8.19 & 16.96 & 22.30 & 53.27 & 51.74 & 33.84 \\
\hline With built-up areas masking & 120.2 & 182.4 & 172.2 & 6.57 & 11.10 & 14.20 & 20.45 & 25.00 & 19.59 \\
\hline
\end{tabular}

